\title{
Revisiting some old results on odd perfect numbers
}

\author{
Jose Arnaldo Bebita Dris ${ }^{1}$ and Doli-Jane Uvales Tejada ${ }^{2}$ \\ ${ }^{1}$ Institute of Mathematics, University of the Philippines \\ Carlos P. Garcia Avenue, Diliman, Quezon City, Philippines \\ e-mails: jbdris@upd. edu.ph, josearnaldobdris@gmail . com \\ ${ }^{2}$ Mathematics Department, College of Natural Sciences and Mathematics \\ Mindanao State University, General Santos City, Philippines \\ e-mail: dolijanetejada@gmail.com
}

Abstract: In this note, we revisit and show how some old results on odd perfect numbers follow from assuming some unproven yet reasonable conjectures.

Keywords: Odd perfect number, Descartes-Frenicle-Sorli conjecture, Dris conjecture, abundancy index, deficiency.

2010 Mathematics Subject Classification: 11A25.

\section{Introduction}

Let $x$ be a natural number. We denote

$$
\sum_{d \mid x} d=\sigma_{1}(x)=\sigma(x)
$$

as the sum of divisors of $x$. Let the abundancy index of $x$ be given by $I(x)=\sigma(x) / x$, and let the deficiency of $x$ be given by $D(x)=2 x-\sigma(x)$. We then have the identity

$$
\frac{D(x)}{x}+\frac{\sigma(x)}{x}=\frac{D(x)}{x}+I(x)=2 .
$$


Furthermore, notice that if $z=\prod_{i=1}^{w} y_{i} t_{i}$ is the prime factorization of $z$, then we have the following formula for the sum of divisors of $z$ :

$$
\sigma(z)=\sigma\left(\prod_{i=1}^{w} y_{i}^{t_{i}}\right)=\prod_{i=1}^{w}\left(\sigma\left(y_{i}^{t_{i}}\right)\right)=\prod_{i=1}^{w} \frac{y_{i}^{t_{i}+1}-1}{y_{i}-1}
$$

where the $y_{i}$ 's are primes and $w=\omega(z)$ is the number of distinct prime factors of $z$. This means that the $\sigma$ function satisfies $\sigma(A B)=\sigma(A) \sigma(B)$ if and only if $\operatorname{gcd}(A, B)=1$, which implies that $\sigma$ is multiplicative.

Therefore, if $\operatorname{gcd}(A, B)=1$, it follows from the formula for $\sigma$ above that

$$
I(A B)=\frac{\sigma(A B)}{A B}=\frac{\sigma(A) \sigma(B)}{A B}=\left(\frac{\sigma(A)}{A}\right) \cdot\left(\frac{\sigma(B)}{B}\right)=I(A) I(B),
$$

which proves that the abundancy index function $I$ is also multiplicative. Finally, notice that the deficiency function $D$ is in general not multiplicative.

We will use, many times, the multiplicativity of the sum-of-divisors function $\sigma$ and the abundancy index function $I$ to prove the results in this paper.

A natural number $N_{1}$ is called perfect if $\sigma\left(N_{1}\right)=2 N_{1}$. (On the other hand, a natural number $N_{2}$ which satisfies $\sigma\left(N_{2}\right)=2 N_{2}-1$ is called almost perfect.) The following result (proved by Euclid and Euler) gives a necessary and sufficient condition for an even natural number $E$ to be perfect.

Theorem 1. An even natural number $E$ is perfect if and only if $E=2^{p-1}\left(2^{p}-1\right)$ for some integer $p$ which makes $2^{p}-1$ prime.

Refer to Dickson [3] to see different proofs of Theorem 1. If a natural number of the form $2^{p}-1$ is prime, then it is called a Mersenne prime. Notice that if $2^{p}-1$ is prime, then $p$ must be prime. (The converse does not hold.) The natural numbers 6, 28, 496, and 8128 are the first four even perfect numbers, and these correspond to the Mersenne primes $2^{p}-1$ with $p$ given by $2,3,5$, and 7 , respectively. It is still unknown if there are infinitely many even perfect numbers. Also, we still do not know if there are odd perfect numbers. It is conjectured that no odd perfect numbers exist.

If $O$ is an odd perfect number, then Euler showed that it must have the form $O=q^{k} n^{2}$, where $q$ is a prime that satisfies $q \equiv k \equiv 1(\bmod 4)$ and $\operatorname{gcd}(q, n)=1$. We will call $q$ the special or Euler prime of $O, q^{k}$ the Euler factor, and $n^{2}$ the non-Euler part. (Notice that both $E$ and $O$ have the forms $N=Q^{K} M^{2}$ where $Q$ is prime, $K \equiv 1(\bmod 4)$, and $\operatorname{gcd}(Q, M)=1$.) Descartes, Frenicle, and more recently Sorli [13] conjectured that $k=1$ holds. Sorli predicts $k=1$ after testing large odd numbers $N^{\prime}$ with $\omega\left(N^{\prime}\right)=8$ for perfection. Subsequently, Beasley [1] reports that "Dickson has documented Descartes' conjecture [that $k=1$ ] as occurring in a letter to Marin Mersenne [on November 15,] 1638, with Frenicle's subsequent observation occurring in 1657”.

Also, according to

1. Ochem and Rao [12], $O>10^{1500}$ and

2. Nielsen [11], $\omega(O) \geq 10$. 
The objective of this paper is to collect some old results on odd perfect numbers which could also be proven by assuming some unproven yet reasonable conjectures. As far as the authors are aware, the approach to these old results presented here is new and has not appeared elsewhere. The novelty of this new approach is that the proofs so produced are significantly shorter than the original arguments.

\section{Summary}

First, we reprove the following result from our previous paper [7] on this topic. We give here a trivial proof of the following lemma.

Lemma 1. If $O=q^{k} n^{2}$ is an odd perfect number with Euler prime q, then

$$
\frac{\sigma\left(O / q^{k}\right)}{q^{k}}=\frac{2 n^{2}}{\sigma\left(q^{k}\right)}=\frac{D\left(n^{2}\right)}{\sigma\left(q^{k-1}\right)}=\operatorname{gcd}\left(n^{2}, \sigma\left(n^{2}\right)\right) .
$$

The proof of Lemma 1 is essentially the same as that contained in [7], except that we make use of an algebraic trick to trim it down.

Dris [4] proved the following result in his M. Sc. thesis. This was eventually published by Dris [6]. (We shall not give an alternative/shorter proof of this theorem here.)

Theorem 2. If $O=q^{k} n^{2}$ is an odd perfect number with Euler prime $q$, then $\sigma\left(O / q^{k}\right) / q^{k} \geq 3$.

Theorem 2 together with Lemma 1 shows that the non-Euler part $n^{2}$ is not almost perfect.

The following theorem was proved by Dris and Luca [9].

Theorem 3. If $O=q^{k} n^{2}$ is an odd perfect number with Euler prime $q$, then $\sigma\left(O / q^{k}\right) / q^{k}>5$.

Remark 1. We note that several authors have made various improvements to the prooffor Theorem 3 in the literature.

We give a short proof of Theorem 3 by assuming the truth of either of the following two conjectures.

Conjecture 1. If $O=q^{k} n^{2}$ is an odd perfect number with Euler prime q, then $k=1$ always holds.

Conjecture 2. If $O=q^{k} n^{2}$ is an odd perfect number with Euler prime q, then the inequality $q^{k}<n$ holds.

In fact, Conjecture 2 implies the following corollary.

Corollary 1. If $O=q^{k} n^{2}$ is an odd perfect number with Euler prime q, then the estimate

$$
\frac{\sigma\left(O / q^{k}\right)}{q^{k}}>\frac{8}{5} n>\frac{8}{5} \sqrt[3]{O}
$$

holds. 
The following (weak) result appeared in [5]. (Observe that the congruence $n^{2}-q^{k} \equiv 0$ (mod 4) holds.)

Lemma 2. If $O=q^{k} n^{2}$ is an odd perfect number with Euler prime $q$, then the inequality $n^{2}-q^{k} \geq$ 8 holds.

We generalize Lemma 2 in the following result, by assuming Conjecture 2 in the "proof".

Theorem 4. If $O=q^{k} n^{2}$ is an odd perfect number with Euler prime $q$ and $n^{2}-q^{k}$ is a power of two, then $n^{2}-q^{k}=2^{2 r+1}$.

In fact, assuming Conjecture 2 is true, we have the following easy corollary.

Corollary 2. If $O=q^{k} n^{2}$ is an odd perfect number with Euler prime q, then the inequality

$$
n^{2}-q^{k}>n^{2}-n=n(n-1)>\sqrt[3]{O}(\sqrt[3]{O}-1)
$$

holds.

Lastly, we prove the following theorem, which follows from Theorem 4.

Theorem 5. If $O=q^{k} n^{2}$ is an odd perfect number with Euler prime $q, n^{2}-q^{k}$ is a power of two, and $q$ is a Fermat prime, then Conjecture 1 is false.

All of the proofs given in this note are elementary. (Note that the proofs of Corollary 1 and Corollary 2 are trivial.)

\section{A trivial proof of Lemma 1}

Let $O=q^{k} n^{2}$ be an odd perfect number with Euler prime $q$. By the definition of perfect numbers, and using the multiplicativity of $I$, we have

$$
2=I(O)=I\left(q^{k} n^{2}\right)=I\left(q^{k}\right) I\left(n^{2}\right)=\left(\frac{\sigma\left(q^{k}\right)}{n^{2}}\right) \cdot\left(\frac{\sigma\left(n^{2}\right)}{q^{k}}\right),
$$

from which it follows that $i(q)=\sigma\left(n^{2}\right) / q^{k}=\sigma\left(N / q^{k}\right) / q^{k}$ is an integer, since $\operatorname{gcd}\left(q^{k}, \sigma\left(q^{k}\right)\right)=$ 1.

Consequently,

$$
i(q)=\frac{\sigma\left(n^{2}\right)}{q^{k}}=\frac{2 n^{2}}{\sigma\left(q^{k}\right)} .
$$

Now, by setting

$$
A=\sigma\left(n^{2}\right), \quad B=q^{k}, \quad C=2 n^{2}, \quad D=\sigma\left(q^{k}\right),
$$

we can use the algebraic identity

$$
\frac{A}{B}=\frac{C}{D}=\frac{C-A}{D-B}
$$


to show that

$$
i(q)=\frac{D\left(n^{2}\right)}{\sigma\left(q^{k-1}\right)}
$$

since $D-B=\sigma\left(q^{k}\right)-q^{k}=1+q+\ldots+q^{k-1}=\sigma\left(q^{k-1}\right)$.

The remaining part is to show that

$$
\operatorname{gcd}\left(n^{2}, \sigma\left(n^{2}\right)\right)=\frac{D\left(n^{2}\right)}{\sigma\left(q^{k-1}\right)}
$$

and this follows easily from

$$
\frac{\sigma\left(n^{2}\right)}{q^{k}}=\frac{2 n^{2}}{\sigma\left(q^{k}\right)}=\frac{D\left(n^{2}\right)}{\sigma\left(q^{k-1}\right)}
$$

and the fact that $\operatorname{gcd}\left(q^{k}, \sigma\left(q^{k}\right) / 2\right)=1$.

This finishes the proof.

\section{A short "proof" of Theorem 3}

Let $O=q^{k} n^{2}$ be an odd perfect number with Euler prime $q$. By Lemma 1, we have

$$
i(q)=\frac{\sigma\left(O / q^{k}\right)}{q^{k}}=\frac{2 n^{2}}{\sigma\left(q^{k}\right)}=\frac{D\left(n^{2}\right)}{\sigma\left(q^{k-1}\right)}=\operatorname{gcd}\left(n^{2}, \sigma\left(n^{2}\right)\right) .
$$

We want to show that, assuming certain unproved yet reasonable conjectures in the literature, then $i(q)>5$.

We claim that $i(q)>q$. The proof of $i(q) \neq q$ is trivial, and follows from $\operatorname{gcd}(q, n)=1$.

We now give two disproofs for $i(q)<q$, by assuming Conjecture 2 and then Conjecture 1 , respectively.

Disproof 1: Assume that Conjecture 2 is true. Then we have $q^{k}<n$ (which implies $q<n$ ), so that

$$
q>i(q)=\frac{\sigma\left(n^{2}\right)}{q^{k}} \text { implies that } \sigma\left(n^{2}\right)<q \cdot q^{k}<n \cdot n=n^{2},
$$

which is a contradiction.

Disproof 2: Suppose that Conjecture 1 holds. Then we have $k=1$, which implies that $q<n$ (a result of Brown [2], albeit still unpublished as of October 2018). We obtain

$$
q>i(q)=\frac{\sigma\left(n^{2}\right)}{q^{k}} \text { implies that } \sigma\left(n^{2}\right)<q \cdot q^{k}=q \cdot q=q^{2}<n^{2},
$$

which, again, is a contradiction.

This ends the short "proof" for Theorem 3.

\section{The "proof" of Theorem 4}

Let $O=q^{k} n^{2}$ be an odd perfect number with Euler prime $q$, and suppose that $n^{2}-q^{k}$ is a power of two. (Assume, for the sake of arriving at a contradiction, that Conjecture 2 is true.) Since 
$q \equiv k \equiv 1(\bmod 4)$, then $q^{k} \equiv 1(\bmod 4)$. Moreover, since $n^{2}$ is a square, then $n^{2} \equiv 1$ $(\bmod 4)$. It follows that $n^{2}-q^{k} \equiv 0(\bmod 4)$. (Note that $q^{k}<n^{2}$ by Theorem 2.) Thus, $n^{2}-q^{k}=2^{t}$ for some integer $t \geq 2$ (by assumption). We shall show that $t$ cannot be even.

To this end, suppose to the contrary that $n^{2}-q^{k}=2^{2 s}$. Then we obtain $n^{2}-2^{2 s}=q^{k}$, from which it follows that

$$
\left(n+2^{s}\right)\left(n-2^{s}\right)=q^{k}
$$

This implies that we have the simultaneous equations

$$
\left\{\begin{array}{l}
n+2^{s}=q^{k-u} \\
n-2^{s}=q^{u}
\end{array}\right.
$$

where $u$ is an integer satisfying $0 \leq u \leq(k-1) / 2$. It follows that we have the system

$$
\left\{\begin{array}{l}
2^{s+1}=q^{k-u}-q^{u}=q^{u}\left(q^{k-2 u}-1\right) \\
2 n=q^{k-u}+q^{u}=q^{u}\left(q^{k-2 u}+1\right)
\end{array} .\right.
$$

We then have

$$
\left\{\begin{array}{l}
u=0,2^{s+1}=q^{k-2 u}-1=q^{k}-1 \\
u=0,2 n=q^{k-2 u}+1=q^{k}+1
\end{array} .\right.
$$

We now consider the Diophantine equation

$$
2^{s+1}=q^{k}-1 .
$$

This can be rewritten as

$$
q^{k}=2^{v}+1
$$

upon setting $v=s+1$. (Note that, since $2 s=t \geq 2$, then $v=s+1 \geq 2$.) We claim that $k=1$. Suppose to the contrary that $k>1$. Then we obtain the only possible solution $(q, v)=(3,3)$ by Mihăilescu's theorem [10]. This contradicts $q \equiv 1(\bmod 4)$. Therefore, $k=1$ holds, and we have

$$
\left\{\begin{array}{l}
u=0,2^{s+1}=q-1 \\
u=0,2 n=q+1
\end{array} .\right.
$$

The second equation implies that $n=(q+1) / 2<q$. This contradicts Conjecture 2, which states that $q^{k}<n$ (and implies that $q<n$ ). (Alternatively, if one would be willing to accept the veracity of the recent preprint [2] by Brown, then we already have a "proof" for the inequality $q<n$. Still other alternative "proofs" are given in the paper by Dris [8] and by Starni [14].)

This finishes the proof. 


\section{The proof of Theorem 5}

Let $O=q^{k} n^{2}$ be an odd perfect number with Euler prime $q$. Suppose that $q$ is a Fermat prime, and that $n^{2}-q^{k}$ is a power of two. By assumption and using Theorem 4, we have

$$
n^{2}-q^{k}=2^{2 r+1}
$$

for some integer $r \geq 2$. In addition, since $q \equiv 1(\bmod 4)$ is a Fermat prime (by assumption), then we can write

$$
q=2^{m}+1
$$

for some integer $m \geq 2$. This means that $m=2^{l}$, for some integer $l \geq 1$. In other words, $q=2^{2^{l}}+1$ is a Fermat prime. Note that it is trivial to prove that

$$
3 \mid 2^{2^{l}-1}+1=\frac{q+1}{2} .
$$

We want to show that $k \neq 1$. To this end, suppose to the contrary that $k=1$. It follows that

$$
n^{2}-q=n^{2}-\left(2^{2^{l}}+1\right)=2^{2 r+1},
$$

from which we obtain

$$
n^{2}-2^{2^{l}}=2^{2 r+1}+1,
$$

which implies that

$$
3 \mid\left(n^{2}-2^{2^{l}}\right)
$$

This means that $3 \nmid n^{2}$, since $l \geq 1$ and $3 \nmid 2^{2^{l}}$.

But we know that $3|(q+1) / 2| n^{2}$. This contradicts $3 \nmid n^{2}$. This finishes the proof of Theorem 5.

\section{Concluding remarks and future research}

Note that, from the inequality $q^{k}<n^{2}$, one can show that $n<q$ implies $k=1$. By the contrapositive, $k>1$ implies $q<n$. Therefore, Theorem 5 implies Theorem 3 (under some additional assumptions). We also get that Theorem 5 implies Theorem 4 (again, under certain additional assumptions). The circular argument can be avoided if an independent proof is established for the inequality $q<n$. This is the subject of the preprint [2], and the papers [14] and [8].

Of course, the arguments presented in the proofs for Theorem 4 and Theorem 5 do not readily generalize to the case when $n^{2}-q^{k}$ is not a power of two, or the case when $q$ is not a Fermat prime. We leave this as a problem for other researchers.

\section{Acknowledgements}

The first author thanks Carl Pomerance, Severino Gervacio, and Timothy Foo for sharing their expertise. The authors are also indebted to the anonymous referees whose valuable feedback helped in improving the overall style and presentation of the manuscript. 


\section{References}

[1] Beasley, B. D. (2013) Euler and the ongoing search for odd perfect numbers, ACMS 19th Biennial Conference Proceedings, Bethel University.

[2] Brown, P. A. (2016) A partial proof of a conjecture of Dris, preprint, https : / / arxiv . org/pdf/1602.01591v1.pdf.

[3] Dickson, L. E. (1971) History of the theory of numbers, Vol. 1, 3-33, Chelsea Pub. Co., New York.

[4] Dris, J. A. B. (2008) Solving the odd perfect number problem: Some old and new approaches, M. S. Math thesis, De La Salle University, Manila, Philippines.

[5] Dris, J. A. B. (2009) Solving the odd perfect number problem: Some new approaches, Electr. Proc. of the 11th Science and Technology Congress, ed. L. Pajo, De La Salle University.

[6] Dris, J. A. B. (2012) The abundancy index of divisors of odd perfect numbers, J. Integ. Seq., 15 (4), Article 12.4.4.

[7] Dris, J. A. B. (2017) Conditions equivalent to the Descartes-Frenicle-Sorli Conjecture on odd perfect numbers, Notes on Number Theory and Discrete Mathematics, 23 (2), 12-20.

[8] Dris, J. A. B. (2017) On a curious biconditional involving divisors of odd perfect numbers, Notes on Number Theory and Discrete Mathematics, 23 (4), 1-13.

[9] Dris, J. A. B., \& Luca, F. (2016) A note on odd perfect numbers, Fibonacci Quart., 54 (4), 291-295.

[10] Mihăilescu, P. (2004) Primary cyclotomic units and a proof of Catalan's Conjecture, J. Reine Angew. Math., 572, 167-195.

[11] Nielsen, P. (2015) Odd perfect numbers, Diophantine equations, and upper bounds, Math. Comp., 84, 2549-2567.

[12] Ochem, P., \& Rao, M. (2012) Odd perfect numbers are greater than $10^{1500}$, Math. Comp., 81, 1869-1877.

[13] Sorli, R. M. (2003) Algorithms in the study of multiperfect and odd perfect numbers, Ph. D. Thesis, University of Technology, Sydney.

[14] Starni, P. (2018) On Dris conjecture about odd perfect numbers, Notes on Number Theory and Discrete Mathematics, 23 (1), 5-9. 